Project Title: Electrically Driven Technologies for Radioactive Aerosol Abatement

Publication Date: June 28, 2001

Lead Principal Investigator:

David W. DePaoli, Chemical Technology Division, Oak Ridge National Laboratory, P.O. Box 2008, Oak Ridge, TN 37831-6224. Phone: (865) 574-6817, e-mail: depaolidw@ornl.gov

Co-Investigator:

Ofodike A. Ezekoye, Department of Mechanical Engineering, University of Texas at Austin, ETC 7.130, Austin, TX 78712. Phone: (512) 471-3085, e-mail: dezekoye@mail.utexas.edu

\title{
Co-Investigators:
}

Costas Tsouris and Valmor F. de Almeida, Chemical Technology Division, Oak Ridge National Laboratory, P.O. Box 2008, Oak Ridge, TN 37831-6224.

\section{Graduate Students:}

Vinayak Barve, Jason Carter, Krishna Lakshminarasimhan and Jonathan Schmidt, University of Texas at Austin Cyrus Riahi-Nezhad, University of Tennessee

\section{RESEARCH OBJECTIVE}

The objective of this research program is to develop an improved understanding of how electrically driven processes, including electrocoalescence, acoustic agglomeration, and electric filtration, may be employed to efficiently treat problems caused by the formation of aerosols during DOE waste treatment operations. The production of aerosols during treatment and retrieval operations in radioactive waste tanks and during thermal treatment operations such as calcination presents a significant problem of cost, worker exposure, potential for release, and increased waste volume. Electrically driven technologies offer promise as remote technologies for improved treatment; however, existing theoretical models are not suitable for prediction and design.

The basis for the project is the general fact that for most particulate collection technology, the marginal collection efficiency increases as the aerosol to be separated increases in size. Using this as a premise, we are investigating mechanisms for increasing the size of particles in an effluent stream as a preprocessing step. Our work is aimed at employing recent advances in theoretical approaches and experimental techniques to improve our understanding of how electrical and acoustic methods may be employed most efficiently alone or in tandem to tackle aerosol problems. The fundamental understanding achieved may provide the basis for development of innovative new approaches and for optimizing removal processes.

\section{RESEARCH PROGRESS AND IMPLICATIONS}

Following is a summary of the work after 33 months of a 3-year project. A coupled experimental/theoretical approach has been undertaken using resources available at UT -Austin and ORNL.

An electro-coalescence unit reactor was built for determining effects of an electric field on the sedimentation of aerosols under controlled quiescent conditions. The apparatus was built, operated, and described in J. Schmidt's master thesis. It was found that the overall sedimentation and coalescence properties of various aerosols tested were quite different and that application of an electric field across the geometry of the unit promoted aggregation/coagulation and enhanced sedimentation. Vinayak Barve, who is pursuing a graduate degree, is currently trying to reproduce Schmidt's experimental results via modeling and simulation of particle coagulation due to Brownian motion and gravitational settling under an electric field.

Past work, undertaken by J. Carter, examined the interaction of particles (10-mm diameter) suspended in a glycerin-filled tank undergoing rigid body oscillatory motion (10 Hz peak-to-peak displacement); the setup was 
aimed at mimicking acoustic effects on aerosols in the visible range. While no appreciable effect was noticed on a pair of settling particles under the effect of gravity in the oscillatory tank, a slurry of particles exhibited delayed sedimentation. The latter is in conflict with studies of agglomeration of particles under acoustic fields. To reconcile these findings, Krishna Lakshminarasimhan, is attempting to use ultrasound to measure the concentration of particles in the tank as a function of elevation. Additional research work is also underway to simulate the behavior of a pair of particles under oscillatory motion; these topics are part of his graduate studies.

An apparatus for measuring the effect of electric fields on flowing aerosols has also been constructed and calibrated. The apparatus allows continuous measurement of aerosol size distribution in the range of 0.4 to 200 micrometers along a 1-m length of a 10-cm square duct using a Malvern Spraytec RTS5000 laser-scattering device. Details on the setup will be available in Cyrus Riahi-Nezhad's master thesis. Encouraging results obtained with water drops of $5-\mu \mathrm{m}$ diameter in air show rates of removal of $70 \%$ in $3.5 \mathrm{~s}$ for air speed of $25 \mathrm{~cm} / \mathrm{s}$ at $22 \mathrm{kV}$. The mechanisms of removal will be illuminated by the next experimental data set planned for this summer at different flow conditions.

Progress has been made developing modeling tools capable of efficiently simulating the interactions of aerosols in applied fields. The basis of the model is the continuous BGK-Boltzmann equation. An implementation based on the discountinuous Galerkin finite element method has been successfully developed and tested for the hyperbolic term of the equation. Tests were made with circular particles in a box ranging from 10 to 1000. The method captures accurately discontinuities in the distribution function. Moreover the computation has been organized in fronts perpendicular to the characteristic directions for unstrutured triangular meshes. The work remaining to be implemented includes the incorporation of collision term, time-integration scheme, and adequate postprocessing of hydrodynamic fields obtained from moments of the distribution function.

Although the work carried out by Schmidt in the electro-coalescence unit reactor indicated enhancement of coalescence of aerosols exposed to electric fields, no quantitative measurement of the size of the coalesced particles were made. Vinayak Barve plans to use the Malvern Spraytec RTS5000 laser-scattering device to further analyze the results obtained in the quiescent conditions. The results of this experiment will be instrumental to explain the prevailing mechanisms of coalescence under flow conditions obtained in the 1-m long duct apparatus.

\section{PLANNED ACTIVITIES}

We plan closely coupled experimental and theoretical work. Experiments will employ the apparatus described above to investigate particle-particle interactions at the fundamental level, and macroscopic particle agglomeration in flowing gas streams under applied fields. The experimental results will be compared with simulations for development and verification of design tools. In parallel with our research work, we will continue to develop contacts with potential end-users. We will attempt to run experiments in our experimental apparatus that match the aerosol characteristics and loading of important potential applications to determine relevance and feasibility for practical implementation.

\section{INFORMATION ACCESS}

Carter, J., and O. A. Ezekoye, " Design of an Oscillating Flow Apparatus for the Study of Low-ReynoldsNumber Particle Dynamics," in press Experiments in Fluids (2000).

Wibowo, Y.W. and Ezekoye, O. A, "Computations of Sedimentation Rates for Acoustically Enhanced Agglomeration," AIChE CCPS Annual International Conference and Workshop on Modeling Consequences of Accidental Releases of Hazardous Materials, San Francisco, CA, September, 1999.

Schmidt, J. J., O. A. Ezekoye, C. K. Riahi-Nezhad, C. Tsouris, V. F. de Almeida, and D. W. DePaoli, "Electrically Driven Technologies for Radioactive Aerosol Abatement," presented at EMSP National Workshop, Atlanta, GA, April 27-27, 2000.

Carter, J. "Study of Fluid Oscillation and its Effect on Low Reynolds Number Particle Sedimentation", M.S. Thesis, The University of Texas at Austin. 2000. 